\title{
钢中白点内表面规则花纹的形成机制
}

\author{
李晓刚 姚治铭 陈 华 柯 伟 \\ (中国科学院腐蚀科学开放实验室, 沈阳 110015)
}

\section{关链词 钢、规则花纹}

结构钢氢含量偏高时,氢原子在缺陷处聚集形成氢分子,发生 $2 \mathrm{H} \rightarrow \mathrm{H}_{2}$ 反应, 在金属内部 形成厚度约为 $10^{-3} \mathrm{~mm}$ 的内裂, 即白点. 白点内充满氢气, 计算表明, 当钢中氢含量为 $4 \mathrm{~mL} /$

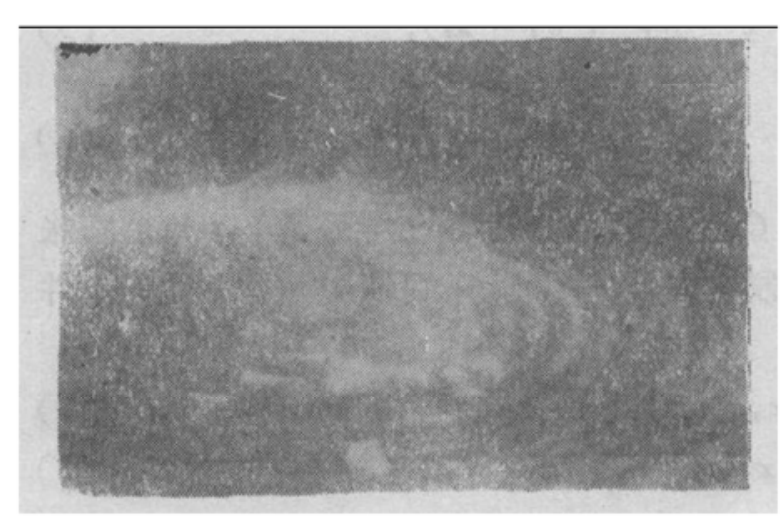

图 1 白点内的规则花纹 SEM

花纹间距为 $1-10 \times 10^{-3} \mathrm{~mm}$ $100 \mathrm{~g}$ 时, 室温的氢压可达 $10^{5} \mathrm{MPa}$. 将白点断口 在电子显微镜下放大到 2000 倍以上, 在白点内表 面上可以观察到一种阶梯状的线条结构, 线条为 彼此相连的同心圆 (图 1) ${ }^{[1]}$. 白点断口上这种规 则花纹, 在结构钢中多有发现, Phillips 用透射电 子显微镜发现过这样的花纹 ${ }^{[2]}$, 陈廉做过系统的 工作.

实验表明,白点并不都带有这种花纹, 只有将 带白点的钢加热后,才能观察到花纹,对带白点钢 热处理是花纹形成条件之一. 目前, 对花纹形成 大致有如下几种解释:（1）凝固时的结晶生长台 阶, (2) 脆性断裂的结果, (3) 凝固后加热时铁原子蒸发或表面扩散生成的台阶. 总之, 都认 为与氢气状态无关.

本文认为花纹形成与白点内高压氢气受热后产生的有序对流有关, 受热后的高压氢产生 了 Benard 现象, 并在白点内表面上刻画出了痕迹. 花纹是高压氢气 Benard 现象的反映, 为 此运用非线性非平衡热力学理论, 计算了高压氢气有序对流的波长. 计算结果与白点内表面 花纹同心圆间距的实测值吻合,并讨论了花纹形成的临界加热条件.

\section{一、规则花纹形成的理论模型}

自 1900 年 Benard 发现流体的六角形有序对流起, 许多研究表明: 流体有序对流花样是 多种多样的,当流体被限制在两块圆形的金属平板间, 对流花样呈旋转状同心圆; 将液态氮放在很小的长方 形盒子中, 施加很小温度梯度, 形成平行旋转状对流转 筒花样 ${ }^{[3]}$.

对本文问题,白点内高压氢气受热后, 氢气开始对

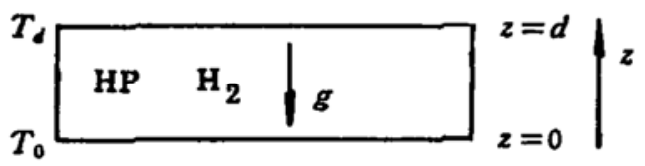

图 2 白点内高压 $\mathrm{H}_{2}$ 研究示意图 流, 起初对流是无序的, 氢气运动符合质量守恒、动量守恒和能量守恒定律:

1991-12-02 收稿，1992-04-07 收修改稿 


$$
\begin{aligned}
& \frac{\partial \rho}{\partial t}+\nabla \cdot(\rho V)+\Delta M_{1}-0, \\
& \frac{\partial \rho V}{\partial t}+\nabla \cdot(\rho V \dot{V}+P-\rho F)+\Delta M_{2}=0, \\
& \frac{\partial \rho_{e}}{\partial t}+\nabla \cdot\left(\rho_{e} V+j_{q}\right)+P: \Delta V+\Delta M_{3}=0,
\end{aligned}
$$

$\Delta M_{1}, \Delta M_{2}$ 和 $\Delta M_{3}$ 分别为白点内反应 $2 \mathrm{H} \rightarrow \mathrm{H}_{2}$ 引起的质量、动量和能量变化, $\rho$ 为密度, $\boldsymbol{V}$ 为流速, $\boldsymbol{P}$ 为应力张量, $\boldsymbol{F}$ 为体力, $\mathrm{e}$ 为内能. 有序对流的出现, 可以看成是上述方程受 到扰动后定态解失去稳定性的结果. 假设氢气饱和, 反应 $2 \mathrm{H} \rightarrow \mathrm{H}_{2}$ 不再发生, $\Delta M_{1}-\Delta M_{2}=$ $\Delta M_{3}-0$, 忽略氢气体积粘滞系数,采用 Koschmider 的简化方法后 ${ }^{[3]}$, 再用扰动法得到扰动 方程

$$
\begin{aligned}
& \nabla \cdot \delta V=0, \\
& \frac{\partial \cdot \delta V}{\partial t}-\frac{\eta \cdot C_{V}}{\lambda}\left(-\nabla \delta P+\nabla^{2} \cdot \delta V+\delta T \cdot Z\right), \\
& \frac{\partial \cdot \delta T}{\partial t}-R \cdot \delta V_{s}+\nabla^{2} \delta T,
\end{aligned}
$$

$R-\rho_{0}^{2} \alpha g\left(T_{0}-T_{1}\right) d^{3} C, / \eta \lambda, \eta$ 为切变粘滞系数, $C$ 、为热容, $\lambda$ 为热传导系数, $\alpha$ 为热膨胀 系数, $g$ 为重力加速度, $\boldsymbol{Z}$ 为单位矢量. 高压氢限制在白点内, 设为不可压缩气体, 边界条件 为

$$
\begin{aligned}
& \delta T(x, y, 0, t)=\delta T(x, y, 1, t)-0, \\
& \delta V(x, y, 0, t)-\delta V(x, y, 1, t)=0, \\
& \frac{\partial \cdot \delta V_{x}}{\partial x}=\frac{\partial \cdot \delta V_{y}}{\partial y}=\frac{\partial \cdot \delta V}{\partial z}=0 .
\end{aligned}
$$

在以上条件下,扰动方程的特征方程为

$$
\omega^{2}+A \omega+B(R, k)-0,
$$

$\omega=0$ 是失稳临界点 ${ }^{[3]}$, 应用 $\omega-0, B(R, k)-0$, 求得 $R_{\mathrm{c}}=1708, k_{\mathrm{c}}-3.117 / d$. 有序对 流特征波长为

$$
l_{c}-2 \pi / k_{c}-2.02 \cdot d .
$$

白点厚度 $d$ 一般为 $10^{-3} \mathrm{~mm}$ 量级, 按(11) 式白点内高压氢气有序对流特征波长为 $1-10 \times$ $10^{-3} \mathrm{~mm}$ ，这与白点内表面规则花纹间距实测值吻合.

$$
\text { 二、讨 论 }
$$

由以上理论模型知, 出现有序对流与外界条件有关, $R>R_{\mathrm{c}}$ 是出现有序对流的条件, 即

$$
\rho_{0}^{2} \cdot \alpha \cdot g \cdot \Delta T \cdot d^{3} \cdot C_{\checkmark} / \eta \lambda>1708 .
$$

实验表明, 只有加热到 $600-700^{\circ} \mathrm{C}$ 以上,白点内表面上才出现规则花纹, 采用 $600^{\circ} \mathrm{C}$ 时氢 气数据进行计算 ${ }^{[4]}, \eta=18 \times 10^{-6} \mathrm{~g} / \mathrm{mm} \cdot \mathrm{s}, \lambda=3.4 \times 10^{-4} \mathrm{~J} / \mathrm{mm} \cdot \mathrm{s} \cdot{ }^{\circ} \mathrm{C}, C,-14.79 \mathrm{~J} /$ $\mathrm{g} \cdot{ }^{\circ} \mathrm{C}$. 设氢气为理想气体, $600^{\circ} \mathrm{C}, 10^{5} \mathrm{MPa}$ 下氢气密度 $\rho-1.38 \mathrm{~g} / \mathrm{mm}^{3}, \alpha-1.67 \times 10^{-3} /$ ${ }^{\circ} \mathrm{C}$, 则

$$
\Delta T \cdot d^{3}>22.7 \times 10^{-9} \mathrm{~mm}^{3} \cdot{ }^{\circ} \mathrm{C}
$$

为临界加热条件. 设某一白点厚度为 $2 \times 10^{-3} \mathrm{~mm}$, 由上式知, 产生规则花纹的外部条作是白 
点上下面温差为 $2.8^{\circ} \mathrm{C}$.

致谢李劲、周向阳助理研究员,陈廉副研究员对本文的有.益讨论,深表感谢。

\section{参考 文 献}

[1] 陈 廉,金属学报, 14(1978), 255 .

[2] Phillips, A., Kerlins, V., Met. Prog., 95(1969), 81.

[3] Koschmider, E. L., Adv. Chem. Phys., 177(1974), 26.

[4]北京石油科学勘探研究院, 如氢裂化与㳖置, 伦学工业出版社, 北京, 1972,172-201 